\title{
Enhancing Safety During a Pandemic Using Virtual Care Remote Monitoring Technologies and UML Modeling
}

\author{
Elizabeth M. Borycki1,2, Andre W. Kushniruk' , Ryan Kletke', Vivian Vimarlund ${ }^{3}$, \\ Yalini Senathirajah ${ }^{4}$, Yuri Quintana ${ }^{5}$ \\ 1 School of Health Information Science, University of Victoria, Canada \\ 2 Michael Smith Foundation for Health Research, Vancouver, Canada \\ 3 Department of Computer and Information Science, Linkoping University, Sweden \\ ${ }^{4}$ Department of Biomedical Informatics, University of Pittsburgh, United States of America \\ 5 Division of Clinical Informatics, Harvard School of Medicine, Harvard University, United States of America
}

\begin{abstract}
Summary
Objectives: This paper describes a methodology for gathering requirements and early design of remote monitoring technology (RMT) for enhancing patient safety during pandemics using virtual care technologies. As pandemics such as COrona Vlrus Disease (COVID-19) progress there is an increasing need for effective virtual care and RMT to support patient care while they are at home.

Methods: The authors describe their work in conducting literature reviews by searching PubMed.gov and the grey literature for articles, and government websites with guidelines describing the signs and symptoms of COVID-19, as well as the progression of the disease. The reviews focused on identifying gaps where RMT could be applied in novel ways and formed the basis for the subsequent modelling of use cases for applying RMT described in this paper. Results: The work was conducted in the context of a new Home of the Future laboratory which has been set up at the University of Victoria. The literature review led to the development of a number of object-oriented models for deploying RMT. This modeling is being used for a number of purposes, including for education of students in health infomatics as well as testing of new use cases for RMT with industrial collaborators and projects within the smart home of the future laboratory. Conclusions: Object-oriented modeling, based on analysis of gaps in the literature, was found to be a useful approach for describing, communicating and teaching about potential new uses of RMT.
\end{abstract}

\section{Keywords}

Remote monitoring technology, assistive living, COVID-19, pandemics, user requirements, safety, public health informatics, health informatics

Yearb Med Inform 2021:264-71

http://dx.doi.org/10.1055/s-0041-1726485

\section{Introduction}

Globally, the COrona VIrus Disease 2019 (COVID-19) has infected 23 million people and the number of infections continues to grow [1-4]. This new, emergent infectious disease has led to significant healthcare and societal costs as well as human suffering. From a healthcare perspective, emergency departments and hospital units have been overwhelmed by the rapid influx of critically ill patients with moderate to severe disease [1-4]. Yet, a significant proportion of the population infected by COVID-19 remain asymptomatic or experience only a mild symptoms of the disease necessitating the need to self-isolate or quarantine to prevent spread to other individuals in our communities [5]. Researchers are attempting to predict the development of COVID-19 symptoms to distinguish between those that develop no, mild, moderate or severe symptoms of disease [5-7]. Such knowledge is necessary for effective health resource planning, allocation and utilization (i.e., deployment and reallocation of human and health technology resources). The approach also allows for tailoring of technology interventions to individual needs; for example, research has illuminated a relationship between COVID-19 severity and sex, race, age, the presence of underlying health conditions (i.e., diabetes, renal disease and chronic pulmonary disease) and socio-economic status [7-9]. To date, there remains a continuum of technology interventions that could be deployed for use in those infected with COVID-19 from sensors that allow for remote monitoring of vital signs and oxygen values in a persons' home through to the pulmonary ventilation of a patient in an intensive care unit (ICU) in a hospital. There is also a need, from a societal perspective, to isolate and quarantine those people who may be infected with COVID-19 while they await the outcome of test results [10]. Lastly, there is a need to maintain population health after an individual has tested positive for the disease, while at the same time ensuring their safety in the home (i.e., should they develop severe symptoms requiring medical intervention and/or immediate hospitalization) $[10,11]$. In such cases there is a need to quarantine to contain the disease by asking individuals to self-isolate in their homes while at the same time being able to engage with caregivers such as family and friends as well as health professionals such as public health officers, telehealth nurses and physicians using virtual care approaches [11-13]. In this paper we aim to describe a methodology for modelling a health technology system to support self-isolation and remote monitoring of individuals at risk for developing COVID-19, or moderate to severe COVID-19 disease. This research aims to fill a gap in the literature on health technology systems requirements modelling [14] for remote monitoring technologies (RMTs) applied to individuals exposed to and/or infected with COVID-19 in the published peer reviewed literature. We begin this paper by discussing the Background and Gaps in the Research Literature. 


\section{Background and Gaps in the Research Literature}

\subsection{Public Health Informatics and COVID-19}

Public health informatics is an important area of research in the sub-field of health or biomedical informatics. From time to time, a new infectious disease or a disease that is currently being controlled emerges and affects significant parts of the human population; for example, SARS-CoV-2 (severe acute respiratory syndrome coronavirus 2), MERS-CoV (Middle East respiratory syndrome-related coronavirus), or Ebola [15]. Mitigation strategies (i.e., self-isolation and quarantine of infected individuals) employed during infectious disease outbreaks have emerged as an important public health strategy that involved collaboration with public health informatics professionals. Here, public health researchers focus on reducing disease spread while public health informatics researchers use technology to develop new ways of isolating and quarantining individuals who have been exposed to or have contracted the disease while at the same time enabling contact with family and health professionals. Such research has been critical to controlling the spread of COVID-19 while at the same time enhancing the safety of the healthy population in a given country jurisdiction (as is the case in our current global COVID-19 pandemic) [5, 10]. One of the most important mitigation strategies in a public health practitioner's toolbox is the act of isolating or quarantining individuals who may be carriers of an infectious disease $[10,11]$. This public health strategy protected populations from possible exposure to the disease and aims to prevent, decrease the number, and size of outbreaks $[10,11]$. With the development of new health technologies used in hospitals and in the community by the individuals, public health informatics has emerged as a technology-oriented discipline aimed at preventing the spread of disease using health technology systems $[10,15,16]$.

\subsection{Public Health Informatics and Health Technology}

In recent years, we have seen a number of health technologies used and repurposed for use to address outbreaks of infectious disease increase in their use by public health informatics professionals, public health officers and government policy makers [15, 16]. For example, jurisdictional and national public health information systems during the SARS-CoV-2 outbreaks had been used to detect and track those affected by new and emergent infectious diseases [16]. Other technologies may have been re-purposed or used in novel ways to focus on addressing outbreak management. Here, re-purposing or novel use of a technology includes: using existing search engines such as Google $\mathbb{R}$ to track searches about specific symptoms, modifying mobile self-assessment and symptom monitoring, customizing electronic health records for tracking and visualization of patient characteristics, symptoms and treatment decisions, and updating decision support systems to reflect real-time research about the disease and its management [15, 16]. This includes using existing consumer health technologies in novel ways to support activities such as social distancing and quarantine to protect those people who are at risk of contracting COVID-19 [15, 17]. Lastly, during a pandemic, new technologies may be deployed to assess their ability to predict population and patient risk, such as machine learning algorithms [18, 19], or to support an individual's decision making such as through a mobile app decision support tool [20], or a web-based Chabot [21]. Under pandemic conditions, existing technologies may be quickly modified and used in novel ways even while new technologies are being developed and implemented to prevent and manage specific aspects of an infectious disease $[15,19,21]$. Therefore, there is a need to understand how existing technologies can be re-purposed and selectively used to develop new technologies that may improve pandemic responses in a strategic manner. Such research is essential to create efficient health technology systems that can be rapidly deployed while at the same time allowing for more fulsome allocation of funding to focus on human resource needs during a pandemic.

\subsection{Pandemics, Health Technology Systems and Unified Modelling Language (UML)}

Health technologies have an important role during pandemics. They allow for dissemination of information to the public about how best to protect oneself. They also allow for widespread dissemination about the presentation, control, mitigation, treatment and management of the new or emergent disease to public health professionals and health professionals $[15,16]$. Technology has been used to effectively disseminate information during past pandemics (e.g., SARS-CoV-2, MERS-CoV, Ebola) $[15,16]$. The COVID-19 pandemic has shown that health technologies can be deployed as public health interventions, helping individuals to self-isolate after exposure to disease and health professionals to remotely monitor those who are positive for the disease in their homes. Unified Modeling Language (UML) is used to develop graphical models of complex, software systems [13,22]. UML is widely used in many phases of software development to draw diagrams, analyze model consistency, create design patterns, generate programming code, and produce reports and documentation. These functions are performed using varying UML tools and to define the ways in which objects interact with each other in varying phases. The majority of this work is focused on software systems rather than technology systems for supporting patients in home settings. To date there have been some published articles on the related topics of use of UML for emergency plan development [23], creation of business processes as part of emergency planning [24], and service design for resilience during public health emergencies [25]. There are also a few published works on use and modeling of multi-agent systems in medicine generally [26] as well as on use of UML modeling in of emerging infectious diseases [27]. However, there has been a lack of articles that have been published with an aim to develop a health technology virtual care system using UML to monitor individuals for and with COVID-19 symptoms [22]. There is a need to define RMT requirements for individuals who have been exposed to and/or infected with COVID-19 
in the peer reviewed literature. Furthermore, the application of UML modelling for use in education and the design and testing of use cases for potential home care applications could have potential benefits for creating sharable and reusable models.

\section{Method}

In our current research the authors developed a method for gathering requirements. We used reviews of the literature and published evidence-based, guidelines to design a health technology system using UML for those individuals, who were exposed to or infected with COVID-19. The health technology system allows for RMT to be used by family and health professionals to monitor affected individuals [10].

\subsection{Setting}

This work took place in a partnership with the University of Victoria (Canada) Smart Home Laboratory in the School of Health Information Science (https://www.uvic.ca/hsd/hinf/ index.php), Canada (AK, EB and RK), the Department of Computer and Information Science (IDA), School of Engineering and Technology at Linköping University, Sweden (VM), the Department of Biomedical Informatics, University of Pittsburgh (YS), U.S.A. and Harvard School of Medicine, Harvard University, U.S.A. (YQ). The Smart Home Laboratory at the University of Victoria was designed to simulate a home environment and allows for studies of the use and usability of a range of health technologies. As part of this research, we also worked with a technology supplier to identify technologies for testing candidate devices and processes that can be used to promote population and individual safety using RMT during a pandemic. Our work was intended to be vendor agnostic so we partnered with a health technology device vendor to identify candidate devices and technologies integrating our knowledge of health technologies, medical science and health systems. The research initially began with the testing of varying devices in a laboratory at the University of Victoria in the School of Health Information Science in
Canada. The lab is currently functional, and we have developed teaching and educational materials for remote use of the technologies as part of our teamwork as well as integrated the methodology into our health informatics curriculum for rapid provincial and country level deployment through trained health informatics professionals.

The lab was accessible for pilot work during the pandemic by the research investigators both in-person and virtually with the addition of virtual collaborative tools such as Zoom ${ }^{\circledR}$ (AK, EB, RK). Public health protocols were followed. The team is currently working remotely as a group using virtual collaborative technologies both within the University of Victoria and with Linköping University, University of Pittsburgh and Harvard University. In addition to this, the researchers have password protected, VPN access to secure drives, and intranet services at the University of Victoria to collaborate on documents (much as we would before social distancing requirements were introduced by the province).

\subsection{Procedure}

We developed our methodology for gathering requirements, designing and testing a system of technologies to be enacted in a series of stages so our approach could be easily re-shared and used by other public health informatics groups. We present our methodology in a series of stages.

\subsection{Stage 1}

We began our work by searching PubMed. gov and the grey literature for articles, and government websites with guidelines describing the signs and symptoms of COVID-19, the progression of the disease and self-isolation as well as quarantine. We specifically focused on sources where there is a description of the following four aspects of COVID-19:

1. Signs and symptoms of COVID-19;

2. The progression of the disease;

3. When there was a need to self-isolate;

4. Who is at risk for developing moderate to severe disease.
This included identification of references [1] through [12] which dealt with the above aspects. In addition to this, we took note of how COVID-19 patients can quickly overwhelm an emergency department during an outbreak and how some patients will be hospitalized so they can receive life-saving treatment. The articles were reviewed by two researchers to identify gaps and unfulfilled patient and health system requirements where RMT could potentially be applied.

\subsection{Stage 2}

In stage 2, the researchers derived use cases, sequence diagrams and design patterns for selecting candidate technologies and identifying objectives for supporting patient self-monitoring, data collection and virtual care [13]. The modelling was based on requirements gathered and extracted from articles and guidelines in Stage 1 (described above). Here, we employed modelling approaches from the health systems analysis and design literature and used object oriented modeling approaches (i.e., with each health technology acting as an object in this new health system). Our reasoning was this: (1) we needed to understand what technologies could be used (including what functions they performed), and (2) how these technologies could be integrated into an existing healthcare system. Published evidence about the disease and our knowledge of public health information systems, telehealth systems and consumer technologies (including differing types of devices that perform the same functions (and can be used interchangeably in a RMT system) was essential to designing the use cases and sequence diagrams. Technologies could be added to the health system, if the patient's symptoms required more intensive monitoring. Technologies could also be removed from the system (i.e., when there was a resolution of some symptoms and a progression towards wellness). Telehealth professionals in conjunction with health informaticists who specialize in public health informatics would assess and determine the system of technologies that would be used and their usage over time as part of the remote monitoring. 


\subsection{Stage 3}

In some cases, individuals testing positive for COVID-19 are also self-isolating, when they were asymptomatic or when experiencing symptoms of the disease [10]. Of note, those infected with COVID-19 may develop no, mild, moderate or severe symptoms, and therefore need RMT. RMT is necessary so that those people who begin to experience moderate to severe symptoms of the disease seek appropriate medical attention in a timely manner [5]. During our current COVID-19 pandemic, it has become essential for individuals to self-monitor their symptoms and to reduce contact with others unless there is a need for health professional intervention (i.e., health service use is necessary only in specific circumstances). Such patient RMT is necessary and appropriate use of health care services by those with COVID-19 and those individuals requiring other urgent treatment for cardiac conditions, cancers ... etc. [5]. In our lab, we began to identify and test RMT that could fulfill the above outlined requirements with a view towards how these technologies would become part of a health technology system for remote monitoring. This involved identifying devices currently available for purchase by consumers, reviewing the functions they could perform and conducting a walkthrough of the device in the context of the Home of the Future. The walkthrough included determining if the device could be used without significant effort by a person who was wearing or using the device to collect health data.

In Table 1, we identify the types of RMT that we identified could be used to monitor a COVID-19 patient based on physiologic needs.

\subsection{Stage 4}

After reviewing several candidate devices that could be used to conduct RMT of COVID-19 patients, we met and collectively designed a health technology system using existing consumer technologies and modeled it using UML. Use case and sequence diagrams were developed and created (see [22] for detailed UML modelling steps). Figure 1 shows requirements for a health technology system in terms of actors and activities using

Table 1 Remote Monitoring Technologies

\begin{tabular}{|l|l|}
\hline Device & Function \\
\hline Thermometer & Monitor for fever and temperature. \\
\hline Blood pressure device & Monitor blood pressure \\
\hline Smart Watch & - Heart rate, Respiration, Pulse Oximeter \\
& - Alerts (e.g., Text, Calls and Emails to \\
& connect with Nurse or physician) \\
& - GPS (e.g., to track compliance with \\
& quarantine) \\
& - Sleep monitor \\
\hline
\end{tabular}

UML. As indicated in the diagram, users (actors) of a system include patients, caregivers (i.e., family, friends and formal caregivers), public health officers, telehealth nurses and researchers. The diagram illustrates a first cut at defining the individual use cases such as summarizing patient data, various forms of measurement and recording of symptoms. Tracking for symptoms would be undertaken through use of vital sign measurement devices (i.e., watch, blood pressure cuff and thermometer), and devices could also include alerts designed for the smart watch for social distancing, information about the number of days into the quarantine period, and educational information focusing on the need to maintain quarantine. Lastly, the device acts as a communication tool for connecting to other devices to collect and send data, voice communication and contact with family, friends, a telehealth nurse and the emergency services (see Figure 1). All of these functions would be performed with access to cellular networks (i.e., the person would not need wireless internet access) as is sometimes the case for older adults and those living in poor socioeconomic circumstances [8].

Figure 2 shows a UML sequence diagram to detail the internal processing involved in one of the activities indicated in the use case diagram - i.e. RMT alerting. We are using these UML sequence diagrams for reasoning about and documenting user requirements, system design processes and potential issues around security and privacy $[28,29]$. This is leading to the development of a library of reusable and shareable design patterns for application in this domain (as UML is an international modeling standard). The researchers identified health technology configurations and methods of implementing the technology in homes as an "in-the-box" health care technology solution in the context of a healthcare system. Knowledge about signs and symptoms of COVID-19 including incubation period and minimum distance needed to stay apart to ensure social distancing was also considered in the design of the technology system. In this case the patient uses a Smartwatch that has an app that allows for RMT. Similar sequence diagrams were created for each of the individual twenty-two use cases (i.e., for each bubble shown in the use case diagram in Figure 1).

\section{Application of the Approach}

We have applied the approach to exploring how a range of devices (e.g., thermometers, pulse oximetry devices, blood pressure cuffs ...etc.) can be integrated into a home alert monitoring system. The use of this in the Smart Home Laboratory has been multi-fold. Firstly, we have used the modelling approach as a basis for teaching upper year undergraduate students and graduate students in health informatics about the potential use and application of home alerting monitoring devices. This work has been the basis for development of prototypes that can then be tested in the lab. In addition, we have extended the use of the UML modelling and subsequent prototype development (based on the mod- 
Borycki et al.



Fig. 1 UML Use Case Diagram of a Health Technology System for Remote Monitoring of COVID-19 Patients to Ensure Patient Safety.

elling) for use in collaborative projects with regional health authorities (developing home monitoring solutions) and a range of vendors of healthcare IT products. The application of UML for this has allowed for improved communication, sharing of design ideas and patterns, and the ultimate advancement of new approaches to remote monitoring. This has had particular importance and impact as the move towards virtual care and home health monitoring has been accelerated by the COVID-19 pandemic.

\section{Conclusions}

In this paper we address a significant gap in the literature, we have created a methodology for designing a health technology system for pandemic response 


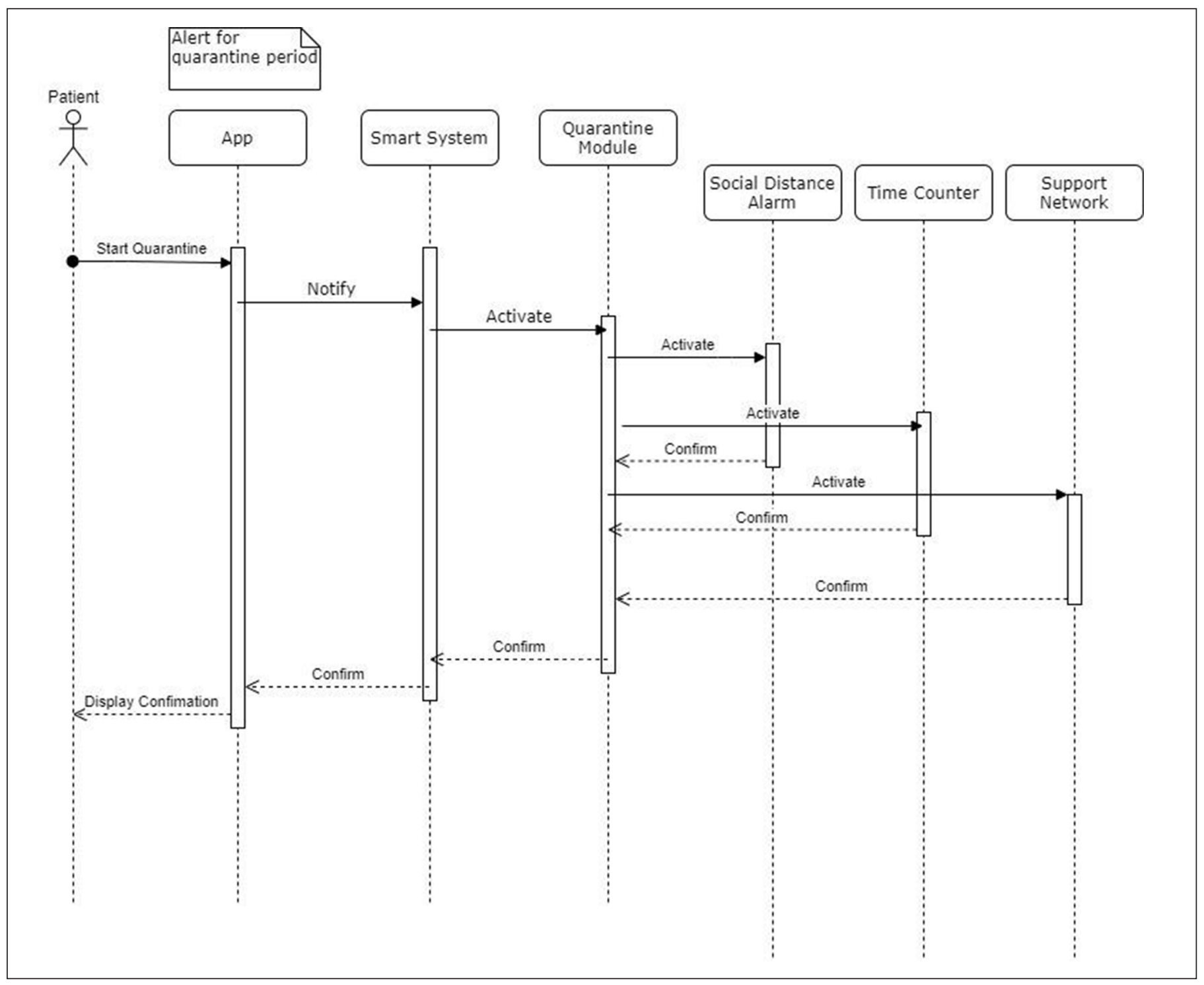

Fig. 2 UML Sequence Diagram for Home Alert Monitoring.

that is based on published articles and guidelines. Much of the response to the COVID-19 has been reactionary. RMT and other technologies used in virtual care have been implemented without much consideration to developing effective and viable health technology systems using UML modelling. In this research we outlined a methodology that can be used to gather requirements and derive use cases, sequence diagrams and design patterns that can be reused across health systems to effectively deploy technologies for RMT of COVID-19. This modeling is now being used to drive the design and implementation of new forms of RMT for testing in the Smart Home Laboratory at the University of Victoria. The approach begins with an evidence-based knowledge of the disease and its progression. This is followed by a focus on pandemic management using health technologies that are currently being used and can be re-purposed for use during a pandemic.
Future research will focus on testing the approach with potential and then actual patients to validate the design pattern for RMT (as a limitation of our current work is the need for validation of the models in real-world contexts). Another limitation of the work in the context of COVID-19 would be that such approaches to RMT would only be used by those who believe there to be a pandemic (i.e., that there is a contagious disease that requires monitoring) [30]. Our future work will include interviewing patients and 
health professionals about their implementation experience and self-monitoring approaches. Data from the interviews will be used to refine the selection of technologies and the implementation approach from a user perspective, vendor perspective and health professional view (i.e. telehealth nurses and physicians). Data collected from participants will include: demographics, eHealth literacy levels, ability to set up the RMT effectively, ability to discuss health issues with a nurse who is reviewing the collected physiologic data online, user experience, and workflow data. As well, health informatics professionals used to support this health technology system will be interviewed about the implementation approach and process of providing care and information technology services respectively.

This research will also be extended to the remote monitoring of vulnerable patients (e.g. cancer, respiratory and cardiovascular disease patients) to prevent deterioration of illness, hospitalization and death during the pandemic. The research could be extended to chronic disease patients during and after the pandemic to improve self-monitoring and self-management of diseases. In addition, we will be exploring applying the approach to include consideration of use of the technologies for people who are entirely asymptomatic, but who wish to have monitoring and alerting available at home to warn at an early stage of physiological indicators (e.g., using pulse oximeter) that are consistent with potential COVID-19 infection (this alerting would lead them to get tested to confirm presence or absence of COVID-19). Our future work will involve creating UML diagrams for RMT for COVID-19 patients with chronic, long term symptoms [31]. We are also currently integrating this approach into health informatics workforce training and building a health informatics workforce to initiate the deployment of the technologies. There is a further need to conduct research on the development of health technology systems to provide support to individuals self-isolating or quarantining in the home. To date, this paper represents a novel application of UML modelling to the development of health technology systems and the development of RMT approaches for COVID-19 modelling.

\section{References}

1. Coronavirus disease (COVID-19) Situation Report - 191. World Health Organization. 2020 Jul 29. Available from: https://www.who.int/ docs/default-source/coronaviruse/situation-reports/20200729-covid-19-sitrep-191.pdf?sfvrsn=2c327e9e_2

2. Guan W, Ni Z, Hu Y, Liang WH, Ou CQ, He JX, China Medical Treatment Expert Group for Covid-19. Clinical Characteristics of Coronavirus Disease 2019 in China. N Engl J Med 2020 Apr 30;382(18):1708-20.

3. Livingston E, Bucher K. Coronavirus Disease 2019 (COVID-19) in Italy. JAMA $2020 \mathrm{Apr}$ 14;323(14):1335

4. Onder G, Rezza G, Brusaferro S. Case-Fatality Rate and Characteristics of Patients Dying in Relation to COVID-19 in Italy. JAMA 2020 May 12;323(18):1775-6.

5. Lauer SA, Grantz KH, Bi Q, Jones FK, Zheng Q, Meredith HR, Azman AS, Reich NG, Lessler J. The incubation period of coronavirus disease 2019 (COVID-19) from publicly reported confirmed cases: estimation and application. Ann Intern Med 2020;172(9):577-82.

6. Bhargava A, Fukushima EA, Levine M, Zhao W, Tanveer F, Szpunar SM, et al. Predictors for Severe COVID-19 Infection. Clin Infect Dis 2020 Nov 5;71(8):19628.

7. Raisi-Estabragh Z, McCracken C, Bethell MS, Cooper J, Cooper C, Caulfield MJ, et al. Greater risk of severe COVID-19 in Black, Asian and Minority Ethnic populations is not explained by cardiometabolic, socioeconomic or behavioural factors, or by $25(\mathrm{OH})$-vitamin D status: study of 1326 cases from the UK Biobank. J Public Health (Oxf) 2020 Aug 18;42(3):451-60.

8. Demenech LM, Dumith SC, Vieira MECD, Neiva-Silva L. Income inequality and risk of infection and death by COVID-19 in Brazil. Rev Bras Epidemiol 2020 Oct 5;23:e200095. Portuguese, English.

9. Vaid A, Somani S, Russak AJ, De Freitas JK, Chaudhry FF, Paranjpe I, et al. Machine Learning to Predict Mortality and Critical Events in a Cohort of Patients With COVID-19 in New York City: Model Development and Validation. J Med Internet Res 2020 Nov 6;22(11):e24018.

10. Anderson RM, Heesterbeek H, Klinkenberg D, Hollingsworth TD. How will country-based mitigation measures influence the course of the COVID-19 epidemic? Lancet 2020 Mar 21;395(10228):931-4.

11. Berekaa MM. Insights into the COVID-19 pandemic: Origin, pathogenesis, diagnosis, and therapeutic interventions. Front Biosci (Elite Ed) 2021 Jan 1;13:117-39.

12. Alhazzani W, Møller MH, Arabi YM, Loeb M, Gong MN, Fan E, et al. Surviving sepsis campaign: guidelines on the management of critically ill adults with Coronavirus Disease 2019 (COVID-19). Read Online: Crit Care Med 2020 Jun;48(6):e440-e469.

13. Whitten JL, Bentley LD, Dittman KC. Systems Analysis and Design Methods 5e. McGraw-Hill
Higher Education; 2000 Aug 1.

14. Hollander JE, Carr BG. Virtually perfect? Telemedicine for covid-19. N Engl J Med 2020 Apr30;382(18):1679-81.

15. Borycki E, Cummings E, Dexheimer JW, Gong Y, Kennebeck S, Kushniruk A, et al. Patient-Centred Coordinated Care in Times of Emerging Diseases and Epidemics. Contribution of the IMIA Working Group on Patient Safety. Yearb Med Inform 2015 Aug 13;10(1):207-15

16. Frisch L E, Borycki EM, Capron A, Mawudeku A, St John, R. Public health informatics in Canada. In: Maguson JA, Fu PC, editors. Public health informatics and information systems (2nd ed.). New York: Springer Verlag; 2013. p. 603-18.

17. Vimarlund V, Borycki E, Kushniruk A, Avenburg $\mathrm{K}$. Ambient assisted living: Identifying new challenges and needs for digital technologies and service innovation. Yearb Med Inform 2021.

18. Peng Y, Li C, Rong Y, Chen X, Chen H. Retrospective analysis of the accuracy of predicting the alert level of COVID-19 in 202 countries using Google Trends and machine learning. J Glob Health 2020 Dec;10(2):020511.

19. Vaid A, Somani S, Russak AJ, De Freitas JK, Chaudhry FF, Paranjpe I, et al. Machine Learning to Predict Mortality and Critical Events in a Cohort of Patients With COVID-19 in New York City: Model Development and Validation. J Med Internet Res 2020 Nov 6;22(11):e24018.

20. British Columbia. SupportApp \& Self-Assessment Tool. Available from: https://bc.thrive.health/

21. Martin A, Nateqi J, Gruarin S, Munsch N, Abdarahmane I, Zobel M, et al. An artificial intelligence-based first-line defence against COVID-19: digitally screening citizens for risks via a chatbot. Sci Rep 2020 Nov 4;10(1):19012.

22. Booch, G. The unified modeling language user guide (2nd Ed.). New York: Addison Wesley; 2005.

23. Zhang Y, Dong C, Zhang X. Description Method of Emergency Plan Base on Multiple Views Framework. In: 2011 International Conference of Information Technology, Computer Engineering and Management Sciences 2011 Sep 24, Vol. 4. IEEE; 2011. p. 160-3.

24. Hamid AH, Rozan MZ, Ibrahim R, Deris S, Rushdi HN, Yunus MM. Understanding and designing business process modelling for emergency plan. In: 2013 International Conference on Research and Innovation in Information Systems (ICRIIS) 2013 Nov 27. IEEE; 2013. p. 564-69.

25. Drăgoicea M, Walletzký L, Carrubbo L, Badr NG, Toli AM, Romanovská F, et al. Service Design for Resilience: A Multi-Contextual Modeling Perspective. IEEE Access 2020 Oct 7;8:185526-43.

26. Hadzic M, Dillon D, Dillon T. Use and Modeling of Multi-agent Systems in Medicine. 2009 20th International Workshop on Database and Expert Systems Application, Linz, Austria, 2009. p. 303-7.

27. Qin H, Shapiro A, Yang L. Emerging Infectious Disease: A Computational Multi-agent Model. 2012 ASE/IEEE International Conference on BioMedical Computing (BioMedCom). Washington, DC, USA; 2012. p. 28-33.

28. Suphakul T, Senivongse T. Development of privacy design patterns based on privacy principles and UML. 2017 18th IEEE/ACIS International Conference on Software Engineering, Artificial 
Enhancing Safety During a Pandemic Using Virtual Care Remote Monitoring Technologies and UML Modeling

Intelligence, Networking and Parallel/Distributed Computing (SNPD), Kanazawa, 2017. p. 369-75.

29. Basso T, Montecchi L, Moraes R, Jino M, Bondavalli A. Towards a UML Profile for Privacy-Aware Applications. 2015 IEEE International Conference on Computer and Information Technology; Ubiquitous Computing and Communications;
Dependable, Autonomic and Secure Computing; Pervasive Intelligence and Computing. Liverpool, UK; 2015. p. 371-8.

30. Mian A, Khan, S. Coronavirus: the spread of misinformation. BMC Med 2020 Mar 18;18(1):89.

31. Nath A. Long-haul COVID. Neurology 2020 Sep 29;95(13):559-60.

\section{Correspondence to:}

Elizabeth Borycki

E-mail: emb@uvic.ca 